\title{
Rapid testing for malaria in settings where microscopy is available and peripheral clinics where only presumptive treatment is available: a randomised controlled trial in Ghana
}

\author{
Evelyn K Ansah, district director of health services, ${ }^{1}$ Solomon Narh-Bana, research officer, ${ }^{1}$ Michael Epokor, \\ research officer, ${ }^{2}$ Samson Akanpigbiam, research officer, ${ }^{2}$ Alberta Amu Quartey, medical superintendent, \\ John Gyapong, director, ${ }^{3}$ Christopher J M Whitty, professor ${ }^{4}$
}

${ }^{1}$ Dangme West District Health Directorate, Dodowa, Ghana

${ }^{2}$ Dodowa Health Research Centre, Dodowa, Ghana

${ }^{3}$ Research and Development Division, Ghana Health Service, Accra, Ghana

${ }^{4}$ London School of Hygiene \& Tropical Medicine, London

Correspondence to: E K Ansah ansahekdr@yahoo.co.uk

Cite this as: $B M J$ 2010;340:c930 doi:10.1136/bmj.c930

\section{ABSTRACT}

Objective To test in West Africa the impact of rapid diagnostic tests on the prescription of antimalarials and antibiotics both where microscopy is used for the diagnosis of malaria and in clinical (peripheral) settings that rely on clinical diagnosis.

Design Randomised, controlled, open label clinical trial. Setting Four clinics in the rural Dangme West district of southern Ghana, one in which microscopy is used for diagnosis of malaria ("microscopy setting") and three where microscopy is not available and diagnosis of malaria is made on the basis of clinical symptoms ("clinical setting").

Participants Patients with suspected malaria.

Interventions Patients were randomly assigned to either a rapid diagnostic test or the current diagnostic method at the clinic (microscopy or clinical diagnosis). A blood sample for a research microscopy slide was taken for all patients.

Main outcome measures The primary outcome was the prescription of antimalarials to patients of any age whose double read research slide was negative for malaria. The major secondary outcomes were the correct prescription of antimalarials, the impact of test results on antibiotic prescription, and the correct prescription of antimalarials in children under 5 years.

Results Of the 9236 patients screened, 3452 were randomised in the clinical setting and 3811 in the microscopy setting. Follow-up to 28 days was $97.6 \%$ (7088/7263). In the microscopy setting, 722 (51.6\%) of the 1400 patients with negative research slides in the rapid diagnostic test arm were treated for malaria compared with 764 (55.0\%) of the 1389 patients in the microscopy arm (adjusted odds ratio $0.87,95 \% \mathrm{Cl} 0.71$ to 1.1 ; $P=0.16)$. In the clinical setting, 578 (53.9\%) of the 1072 patients in the rapid diagnostic test arm with negative research slides were treated for malaria compared with 982 (90.1\%) of the 1090 patients with negative slides in the clinical diagnosis arm (odds ratio $0.12,95 \% \mathrm{Cl} 0.04$ to $0.38 ; \mathrm{P}=0.001)$. The use of rapid diagnostic tests led to better targeting of antimalarials and antibiotics in the clinical but not the microscopy setting, in both children and adults. There were no deaths in children under 5 years at 28 days follow-up in either arm.

Conclusion Where microscopy already exists, introducing rapid diagnostic tests had limited impact on prescriber behaviour. In settings where microscopy was not available, however, using rapid diagnostic tests led to a significant reduction in the overprescription of antimalarials, without any evidence of clinical harm, and to better targeting of antibiotics.

Trial registration ClinicalTrials.gov NCT00493922.

\section{INTRODUCTION}

Falciparum malaria is one of the most important diseases in Africa, both in terms of mortality and burden on health services. Many people in Africa who have malaria are not being treated with effective drugs. ${ }^{1}$ Simultaneously, a substantial proportion, sometimes the majority, of those treated for malaria in both East and West Africa are not actually infected with malaria parasites. $^{2-4}$ Given that malaria is the most common diagnosis made in African children and one of the most common in adults, both underdiagnosis and overdiagnosis of malaria have substantial public health implications. Missing true cases threatens the lives of otherwise well children, whereas people wrongly treated for malaria often have alternative diagnoses-especially bacterial diseases, some of which are potentially fatal - that are not being treated..$^{56}$

Overdiagnosis also leads to overuse of antimalarial drugs, potentially increasing the risk of spread of drug resistant malaria. Given that the current highly effective drugs are substantially more expensive than previous antimalarials, the economic implications of overdiagnosis are considerable and undermine the cost effectiveness of the newer artemisinin based combination therapies (ACTs). ${ }^{7}$ Malaria incidence is falling in some areas, and the problem of overtreatment becomes more important where malaria incidence is decreasing because the proportion of serious causes of febrile illness that are not malaria increases. ${ }^{8}$ Overdiagnosis of malaria is therefore likely to be an increasing problem. 


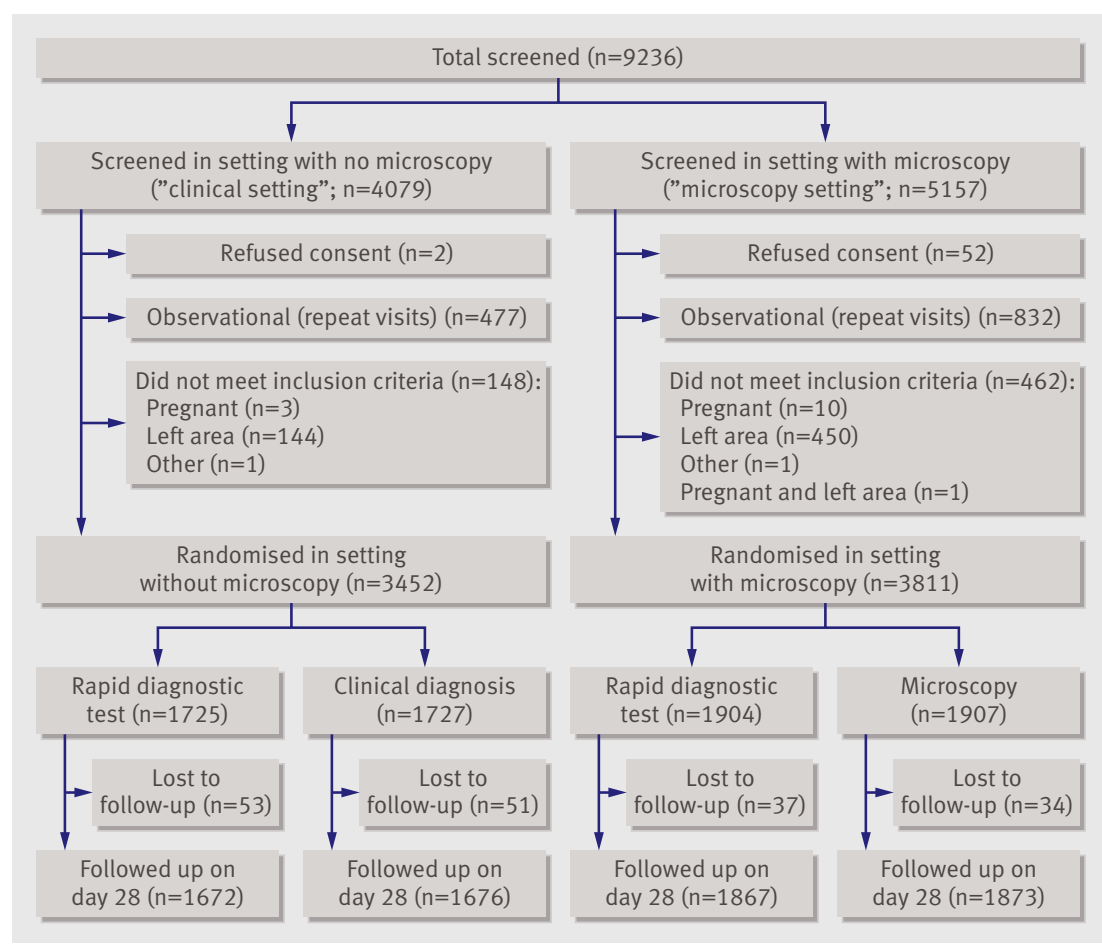

Trial profile for rapid diagnostic tests versus microscopy or clinical diagnosis in two settings in Ghana

Until recently, proper diagnosis of malaria relied on light microscopy. This approach is possible or cost effective only in settings with high throughput and where trained microscopists are available. ${ }^{9}$ New rapid diagnostic tests (RDTs) for falciparum malaria are sensitive and specific (although there is marked variation between different makes of tests), ${ }^{11}$ and can be used in peripheral settings (rural and community settings) where most patients in Africa get their care. They do not require microscopes, electricity, or trained laboratory staff. They are also potentially cost effective, but only if prescribers use the results to guide their prescribing. ${ }^{12}$ Studies in central clinics in East Africa where microscopy is used for diagnosis suggest that clinicians often do not change their prescribing behaviour when given rapid diagnostic tests to use, ${ }^{13}{ }^{14}$ and one study indicated that rapid diagnostic tests even have the potential to be harmful by encouraging undertreatment of true malaria compared with presumptive treatment. ${ }^{15}$ A good response to the results of rapid diagnostic tests has, however, been achieved under certain circumstances. ${ }^{1617}$

Several major questions need to be answered about how best to use rapid diagnostic tests for the management of febrile illness in Africa. The most important of these questions relates to the role of rapid diagnostic tests in settings where microscopy is not available, because most children and adults in Africa seek care in such settings. Inaccurate prescription of antimalarials is greater in such situations because causes of febrile illness are difficult to differentiate clinically in the absence of tests. Testing the impact of rapid diagnostic tests in this setting is therefore essential. There is much hope, but limited evidence, that they will also have an impact where microscopy is not available because prescribers who have not become used to ignoring microscopy results because microscopy is not available may be much more likely to use rapid diagnostic tests to guide diagnosis and treatment. Additionally, most published studies on the impact of rapid diagnostic tests on management of febrile illness were performed in East Africa, but the largest burden of febrile diseases is in the populous countries of West Africa, where malaria epidemiology, clinical training, and the structure of the health service are very different from in East Africa. Finally, there are few follow-up data on patients once they have left clinics, and one of the concerns is that rapid diagnostic tests may lead to greater mortality and morbidity because they lead to cases of true malaria being missed.

We set out to study the impact of rapid diagnostic tests on prescribing of antimalarials and antibiotics in West Africa in two settings serving the same population: one where microscopy is routinely available (to allow comparison with previous studies) and the other where microscopy is not available (peripheral clinics, which are representative of the majority of clinics in sub-Saharan Africa). Most deaths from malaria are in children, but the majority of antimalarials in Africa are consumed by adults, so we examined the impact in both children and adults.

\section{METHODS}

The study was a randomised, controlled, open label clinical trial carried out in the Dangme West district of southern Ghana, a rural district with an estimated population of about 130000 living in scattered communities of less than 2000 people. The district has four health centres and six community based clinics. There is one publicly owned laboratory based in Dodowa Health Centre, Dodowa, and two privately owned laboratories in two other sub districts. All health facilities where there are no laboratories rely on clinical diagnosis. The district has no hospital.

In 2008, a total of 31971 cases of malaria were reported in all health facilities in the district, both public and private, which accounted for approximately $41 \%$ of all reported attendances. Of these 31971 cases, $7808(24 \%)$ were in children less than 5 years of age. A high proportion of these diagnoses were presumptive (that is, made without tests).

\section{Setting}

The trial was undertaken in four health facilities in the district, one of which (Dodowa Health Centre) had facilities for microscopy. The others, where the diagnosis was normally presumptive, included one clinic that is privately owned. The Dodowa Health Centre is subsequently referred to as the "microscopy setting," whereas the other health facilities as a group are referred to as the "clinical setting."

The healthcare professionals were mostly nurses with two years of basic training followed by several years of experience in hospitals, or nurses with three 
Table 1|Baseline characteristics of patients randomly allocated to rapid diagnostic testing or to microscopy or clinical diagnosis

\begin{tabular}{|c|c|c|c|c|}
\hline & \multicolumn{2}{|c|}{ Health centre with microscopy ("microscopy setting") } & \multicolumn{2}{|c|}{ Health facilities with no microscopy ("clinical setting") } \\
\hline & Rapid diagnostic test arm & Microscopy arm & Rapid diagnostic test arm & Clinical diagnosis arm \\
\hline Median age (months (IQR)) & $14(4$ to 33$)$ & 13 (4 to 31$)$ & 12 (4 to 32 ) & 12 (4 to 30$)$ \\
\hline Proportion female & $1148 / 1904(60.3)$ & $1155 / 1907(60.6)$ & $1035 / 1725(60.0)$ & $999 / 1727$ (57.9) \\
\hline $\begin{array}{l}\text { Proportion belonging to bottom two wealth quintiles } \\
\text { (out of study participants who were successfully followed up) }\end{array}$ & $745 / 1867(40.0)$ & $734 / 1873(39.0)$ & $634 / 1672(38.0)$ & $687 / 1676(41.0)$ \\
\hline Median temperature ${ }^{\circ} \mathrm{C}$ (interquartile range) & 36.5 (36 to 37.4$)$ & 36.5 (36 to 37.4 ) & 37.2 (36.2 to 38.4$)$ & $37.2(36.3$ to 38.4$)$ \\
\hline Proportion with fever in past 48 hours & $1018 / 1904(53.5)$ & $1002 / 1907(52.5)$ & $919 / 1725(53.3)$ & $937 / 1727(54.3)$ \\
\hline Proportion positive for malaria parasites on double read slide & $498 / 1904$ (26.2) & $513 / 1907$ (26.9) & $651 / 1725(37.7)$ & $634 / 1727(36.7)$ \\
\hline Parasite count per $\mu \mathrm{l}(\mathrm{IQR})$ in slide positive patients & 9200 (1620 to 34640$)$ & 8000 (1360 to 27200$)$ & 9680 (1500 to 28760$)$ & 10240 (1640 to 29600$)$ \\
\hline
\end{tabular}

Values given as number (percentage) unless otherwise indicated.

years of basic training resulting in a diploma and usually a number of years of experience in hospitals. The healthcare professionals also included auxiliary nurses with two years of basic training and additional "on the job" training in basic curative care. The final category of healthcare professionals was nurse practitioners with three years of basic training resulting in a diploma followed by an additional two years of training in curative care, often separated by a number of years of work in hospitals and sometimes training in midwifery. The latter group was the most highly trained and experienced. There was only one doctor.

Before the study begun, all healthcare professionals in participating centres were given identical training about the sensitivity and specificity of rapid diagnostic tests, alternative causes of febrile illness, and the Ghana national guidelines, which indicate presumptive treatment for children under 5 years of age (that is, the treatment is assigned without carrying out a diagnostic test). The first day focused on differential diagnoses of fever in children and adults, whereas the second day focused on the study itself and rapid diagnostic tests. In the training, emphasis was placed on the difference between a diagnosis by microscopy, which depends on identification of the malaria parasite, and a diagnosis according to the rapid diagnostic test, which relies on an antigen-antibody reaction. This distinction was emphasised to improve the perceptions of negative clinical slide results among healthcare professionals. The healthcare professionals were also trained to carry out and interpret the rapid diagnostic test themselves. They were, however, left free to make their own clinical decisions after the initial training. The training took two days and was designed to replicate the best training that could reasonably be delivered in a routine roll-out of rapid diagnostic tests.

Participants and randomisation

All patients visiting the health facilities were screened for enrolment into the study. The inclusion criteria were that the healthcare professional considered treating the patient for malaria and wanted to test for malaria or treat the patient with an antimalarial. Exclusion criteria were pregnancy, illness severe enough to warrant referral to a hospital, insistence by the healthcare professional on a particular test or a particular method of treatment, patient insistence on a particular test, refusal of consent by the patient or guardian (for minors), not living in the district or nearby, or not intending to remain in the district for the next two months for follow-up.

OptiMAL-IT rapid diagnostic test kits (Diamed AG, Cressier sur Morat, Switzerland) ${ }^{18}$ were used for this study. This test uses a dipstick coated with monoclonal antibodies against plasmodium lactate dehydrogenase, which is released from erythrocytes infected with the parasite. Local microscopy used Giemsa staining, with the parasite count recorded as,+++ , or +++ .

In both settings a blood sample for a research slide was taken for all patients. Research slides were Giemsa stained and read by two independent microscopists who were blind to the study allocations and results of the rapid diagnostic tests. In cases where the two readers had discordant results, the slide was read by a third reader. These analyses by the expert microscopists were undertaken later and not used by the healthcare professionals in their treatment decisions. The research slides were examined under $100 \times$ oil immersion magnification and a film was recorded as positive or negative and parasite count recorded for malaria on the basis of the number of asexual parasites per 200 white blood cells.

Randomisation was carried out using Stata 8 software (Statacorp, College Station, TX) by staff not involved in clinical care. Randomisation was carried out in blocks of 10 for each setting. The allocations were placed in sealed, sequentially numbered, opaque envelopes and delivered to the study team. At all health facilities, envelopes were arranged and allocated sequentially.

\section{Intervention}

At the single health facility with microscopy facilities, patients who fulfilled the inclusion criteria and who the health professional wished to have tested for malaria were sent to a member of the study team, who carried out an evaluation for eligibility and obtained informed consent and demographic and clinical data. A sealed, sequentially numbered envelope containing the patient's test allocation - either "rapid diagnostic test" or "microscopy"-was opened in the presence of the patient; this constituted entry to the trial and intention to treat analysis was conducted on the basis of this allocation. The allocated test, either rapid diagnostic test or 
Table $2 \mid$ Antimalarial and antibiotic prescribing behaviour measured against the double read research slides*

\begin{tabular}{|c|c|c|c|c|}
\hline & \multicolumn{2}{|c|}{$\begin{array}{l}\text { Health centre with microscopy } \\
\text { ("microscopy setting") }\end{array}$} & \multicolumn{2}{|c|}{$\begin{array}{l}\text { Health facilities with no microscopy } \\
\text { ("clinical setting") }\end{array}$} \\
\hline & $\begin{array}{l}\text { Rapid diagnostic } \\
\text { test arm }\end{array}$ & $\begin{array}{c}\text { Microscopy } \\
\text { arm }\end{array}$ & $\begin{array}{l}\text { Rapid diagnostic } \\
\text { test arm }\end{array}$ & $\begin{array}{c}\text { Clinical diagnosis } \\
\text { arm }\end{array}$ \\
\hline Proportion of patients with a positive research slide treated with antimalarials & $462 / 496(93.2)$ & $458 / 511(89.6)$ & $626 / 647(96.8)$ & $616 / 633(97.3)$ \\
\hline Proportion of patients with a negative research slide treated with antimalarials & $722 / 1400(51.6)$ & $764 / 1389(55.0)$ & $578 / 1072(53.9)$ & $982 / 1090(90.1)$ \\
\hline Proportion of patients with a positive research slide treated with antibiotics & $67 / 496(13.5)$ & $67 / 511(13.1)$ & $87 / 647(13.4)$ & $102 / 633(16.1)$ \\
\hline Proportion of patients with a negative research slide treated with antibiotics & $374 / 1400(26.7)$ & $383 / 1389(27.6)$ & $370 / 1072(34.5)$ & $282 / 1090(25.9)$ \\
\hline Correct treatment of malaria & $1140 / 1896(60.1)$ & $1085 / 1900(57.1)$ & $1123 / 1719(65.3)$ & $724 / 1723(42.0)$ \\
\hline
\end{tabular}

Values given as number (percentage) unless otherwise indicated.

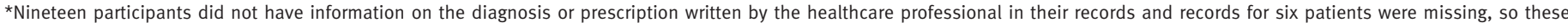
individuals were excluded from the analyses.

local microscopy, was then carried out in the clinic's laboratory, with a separate research slide taken for all cases to be read later. The laboratory results were written out as usual in the case of clinical microscopy, and rapid diagnostic test results were recorded and the dipstick sent in its case to the healthcare professional to read as well.

At the three health facilities where microscopy was not available, healthcare professionals sent patients in whom they suspected malaria to the study team. Informed consent was sought and eligible patients were randomised in the same way as those at the clinic with microscopy facilities. In the case of allocation to the rapid diagnostic test group, the test was carried out by a member of the study team and the results recorded. The results were sent together with the rapid diagnostic test kit to the healthcare professional for clinician read diagnosis and subsequent treatment. In cases where the patient was assigned to clinical diagnosis, the laboratory results form with "clinical diagnosis" ticked on it was returned to the healthcare professional. A blood sample for a research slide was also taken - this was not read until later.

\section{Outcome measures}

The primary outcome was the proportion of patients, either children or adults, who did not have malaria on the basis of a negative double read research slide but were prescribed antimalarial treatment.

Secondary outcomes included the proportion of patients whose research slide was positive for malaria and who were not prescribed an antimalarial, the proportion of patients whose research slide was either positive or negative who were prescribed antibiotics, the proportion of patients whose rapid diagnostic test result was positive and who were not prescribed an antimalarial, and the composite measure of "correct prescription of antimalarials," meaning antimalarials being given to all patients who were test positive and not to anyone who was test negative, in all patients and in children under 5 years only. The sensitivity and specificity of rapid diagnostic tests were also calculated against the double read research slides. The response of healthcare professionals was measured objectively by appropriate prescription of an effective antimalarial or a broad spectrum antibiotic capable of treating common
Gram positive or Gram negative pathogens (for example, pneumococcus or non-typhoid salmonella).

Patients were followed up at their homes 28 days after enrolment to determine subsequent treatment seeking and any mortality or admissions to hospital, and to confirm socioeconomic variables. A random sample of 500 patients in both the clinical and microscopy settings had blood films taken during follow-up.

\section{Statistical analysis}

Data were entered into Epi Info 6 (Centers for Disease Control and Prevention, Atlanta, GA) and analysed using Stata 10 (Statacorp). Simple proportions were calculated for the study groups overall, and then results were stratified by age. In all cases results from the microscopy setting and those from the clinical setting were analysed separately. For the primary and major secondary outcomes, odds ratios were calculated in an uncorrected logistic regression model and subsequently adjusted for age, sex, socioeconomic status, and clustering by healthcare professional. Socioeconomic status was determined by constructing an asset index, which has been validated locally, and calculating quintiles. ${ }^{19}$ Clustering by healthcare professional was adjusted for by using the "svy" command in Stata, with healthcare professionals as the primary sampling unit.

The sample size was calculated to allow us to detect a $25 \%$ reduction in overprescription of antimalarials in patients of any age in the microscopy and clinical settings independently $(\alpha=0.05, \beta=0.8)$; a reduction less than this was thought unlikely to lead to a public health change as large and potentially expensive as deploying rapid diagnostic tests. Published studies suggest that an antimalarial will be prescribed in at least $45 \%$ of patients who have a negative blood slide result, where microscopy is present. The primary outcome was overprescription irrespective of age in each setting independently (that is, both in the clinical setting and the microscopy setting), but we wished to have power for a major secondary analysis restricted to children, so the trial was powered for this analysis (and, therefore, overpowered for the primary analysis). We assumed on the basis of previous clinic data that the ratio of patients in whom malaria was suspected would be $2: 1$ for children to adults. In adults we conservatively estimated that overprescription would be $50 \%$. In children we 
Table 3 Antimalarial and antibiotic prescribing in patients randomly allocated to rapid diagnostic testing or clinical diagnosis in health facilities with no microscopy and in patients assigned rapid diagnostic testing or microscopy in a health centre with microscopy*

\begin{tabular}{|c|c|c|c|c|}
\hline & \multicolumn{2}{|c|}{$\begin{array}{l}\text { Health centre with microscopy } \\
\text { ("microscopy setting") }\end{array}$} & \multicolumn{2}{|c|}{$\begin{array}{l}\text { Health facilities with no microscopy } \\
\text { ("clinical setting") }\end{array}$} \\
\hline & $\begin{array}{l}\text { Rapid diagnostic } \\
\text { test arm }\end{array}$ & $\begin{array}{l}\text { Microscopy } \\
\text { arm }\end{array}$ & $\begin{array}{l}\text { Rapid diagnostic } \\
\text { test arm }\end{array}$ & $\begin{array}{l}\text { Clinical diagnosis } \\
\text { arm }\end{array}$ \\
\hline Proportion of patients treated with antimalarials only & $1018 / 1896(53.7 \%)$ & $1038 / 1900(54.6 \%)$ & $993 / 1719(57.8 \%)$ & $1268 / 1723(73.6 \%)$ \\
\hline Proportion treated with antibiotics only & $275 / 1896(14.5 \%)$ & $267 / 1900(14.1 \%)$ & $246 / 1719(14.3 \%)$ & $54 / 1723(3.1 \%)$ \\
\hline Proportion treated with both antimalarials and antibiotics & $166 / 1896(8.8 \%)$ & $183 / 1900(9.6 \%)$ & $211 / 1719(12.3 \%)$ & $330 / 1723(19.2 \%)$ \\
\hline $\begin{array}{l}\text { Proportion of patients with a positive rapid diagnostic test or clinic microscopy result } \\
\text { treated with antimalarials }\end{array}$ & $587 / 598(98.2 \%)$ & $565 / 575(98.3 \%)$ & $703 / 706$ (99.6\%) & NA \\
\hline $\begin{array}{l}\text { Proportion of patients with a negative rapid diagnostic test or clinic microscopy } \\
\text { result treated with antimalarials }\end{array}$ & $597 / 1298(46.0 \%)$ & $656 / 1325(49.5 \%)$ & $501 / 1013(49.5 \%)$ & NA \\
\hline $\begin{array}{l}\text { Proportion of patients with a positive rapid diagnostic test or microscopy result } \\
\text { treated with antibiotics }\end{array}$ & $67 / 598(11.2 \%)$ & $71 / 575$ (12.3\%) & $102 / 706(14.4 \%)$ & NA \\
\hline $\begin{array}{l}\text { Proportion of patients with a negative rapid diagnostic test or microscopy result } \\
\text { treated with antibiotics }\end{array}$ & $374 / 1298(28.8 \%)$ & $379 / 1325(28.6 \%)$ & $355 / 1013(35.0 \%)$ & NA \\
\hline
\end{tabular}

estimated it would be $30 \%$. Detecting a $25 \%$ reduction in overprescription required a sample size of 2500 children and 1000 adults, or 3500 patients overall for the microscopy setting. In the clinical setting, we estimated that the overprescription of antimalarials was likely to be $10 \%$ higher in both children and adults; thus around 3000 patients were required. The total required sample size for the whole study was therefore 6500 .

This trial was prospectively registered at ClinicalTrials.gov as NCT00493922.

\section{RESULTS}

The trial ran from 6 August 2007 to 24 December 2008. Of the 9236 patients screened, 3452 were randomised in the clinical setting (1725 patients to the rapid diagnostic test and 1727 to clinical diagnosis) and 3811 in the microscopy setting (1904 patients to the rapid diagnostic test and 1907 to microscopy). Final data for the primary outcome were not available in nine patients whose records were lost $(0.1 \%)$. A total of 175 patients $(2.41 \%)$ were lost to follow-up, with no obvious difference between those lost and the rest of the study group.

There were a total of 16 and 13 prescribers in the microscopy and clinical settings, respectively, over the period of the study. Three healthcare professionals were transferred out of the district, one of whom retired, and two new health professionals, including a doctor, were transferred in during the latter part of the study. The median number of patients seen was 121 (range 3 to 1450). The highest number was by the sole healthcare professional at the private clinic without microscopy.

The figure shows the flow of patients through the trial, and baseline characteristics of the randomised patients in the two settings are shown in table 1.

Judged against the diagnoses according to the double read research slide, rapid diagnostic tests did not lead to a reduction in incorrect prescribing of antimalarials in the microscopy setting, or to a change in antibiotic prescribing. The proportion of patients with a negative research slide wrongly treated with antimalarials was $51.6 \%(722 / 1400)$ in the rapid diagnostic test arm and $55.0 \%(764 / 1389)$ in the microscopy arm (odds ratio (OR) 0.88, 95\% CI 0.75 to 1.02 ; table 2).

There was likewise no difference in prescribing once the analysis was adjusted for the predefined potential confounding factors of age, sex, socioeconomic status, and clustering by healthcare professional (adjusted OR $0.87,95 \% \mathrm{CI} 0.71$ to $1.1 ; \mathrm{P}=0.16)$. Among patients with a positive research slide, $6.9 \%(34 / 496)$ in the rapid diagnostic test arm were not treated for malaria compared with $10.4 \%$ (53/511) in the microscopy arm. The odds ratio of rapid diagnostic tests leading to better targeting of antimalarials in the microscopy settingdefined as all individuals positive for malaria according to their research slide being treated for malaria and all negative cases not being treated - was 1.1 (95\% CI 0.99 to $1.3, \mathrm{P}=0.05$; adjusted OR $1.2,95 \%$ CI 0.94 to 1.4 ). There was no evidence of clustering by healthcare professional having more than a minor effect. Rapid diagnostic tests had no clinically relevant impact on antibiotic prescribing in this setting: $23.3 \%(441 / 1896)$ in the rapid diagnostic test arm and 23.7\% (450/1900) in the microscopy arm were treated with antibiotics.

On the other hand, rapid diagnostic tests had a significant impact on the accuracy of prescribing in the clinical setting (where microscopy was not available). The proportion of patients with negative research slides wrongly treated with antimalarials was $53.9 \%$ (578/1072) in the rapid diagnostic test arm compared with $90.1 \%(982 / 1090)$ in the clinical diagnosis arm (OR 0.13, 95\% CI 0.01 to 1.6; adjusted OR 0.12, 95\% CI 0.04 to $0.38 ; \mathrm{P}=0.001$ ). The proportion of patients with a positive slide not treated for malaria was similar in the two arms: $2.7 \%(17 / 633)$ in the clinical arm and $3.2 \%(21 / 647)$ in the rapid diagnostic test arm. The odds ratio of rapid diagnostic tests leading to better targeting of antimalarials in this setting was $2.6(95 \%$ CI 2.2 to 3.0). When adjusted for age, sex, socioeconomic status and clustering by healthcare professional, 
Table $4 \mid$ Antimalarial and antibiotic prescribing by age group in patients randomly allocated to rapid diagnostic testing or clinical diagnosis in health facilities with no microscopy and in patients assigned rapid diagnostic testing or microscopy in a health centre with microscopy*

\begin{tabular}{|c|c|c|c|c|}
\hline & \multicolumn{2}{|c|}{$\begin{array}{l}\text { Health centre with microscopy } \\
\text { ("microscopy setting") }\end{array}$} & \multicolumn{2}{|c|}{$\begin{array}{l}\text { Health facilities with no microscopy } \\
\text { ("clinical setting") }\end{array}$} \\
\hline & Rapid diagnostic test arm & Microscopy arm & Rapid diagnostic test arm & Clinical diagnosis arm \\
\hline \multicolumn{5}{|c|}{ Treated with antimalarials } \\
\hline Up to 1 year & $57 / 101(56.4)$ & $50 / 87(57.5)$ & 45/61 (73.8) & $51 / 58(87.9)$ \\
\hline $1-5$ years & $330 / 469(70.4)$ & $343 / 482(71.2)$ & $370 / 458(80.8)$ & 458/492 (93.1) \\
\hline $5-15$ years & $329 / 442(74.4)$ & $344 / 475(72.4)$ & $356 / 422(84.4)$ & $391 / 410(95.4)$ \\
\hline $16-70$ years & $427 / 787(54.3)$ & $444 / 767$ (57.9) & $401 / 719(55.8)$ & $647 / 707$ (91.5) \\
\hline 70 years or above & 41/97 (42.3) & 40/89 (44.9) & $32 / 59(54.2)$ & $51 / 56(91.1)$ \\
\hline \multicolumn{5}{|c|}{ Treated with antibiotics } \\
\hline Up to 1 year & 41/101 (40.6) & $35 / 87(40.2)$ & $25 / 61(41.0)$ & $17 / 58(29.3)$ \\
\hline $1-5$ years & $117 / 469(25.0)$ & $119 / 482(24.7)$ & $126 / 458(27.5)$ & $140 / 492(28.5)$ \\
\hline $5-15$ years & $85 / 442(19.2)$ & $88 / 475(18.5)$ & $91 / 422(21.6)$ & $77 / 410(18.8)$ \\
\hline $16-70$ years & $181 / 787(23.0)$ & $187 / 767(24.4)$ & $201 / 719(28.0)$ & $144 / 707(20.4)$ \\
\hline 70 years or above & $17 / 97(17.5)$ & $21 / 89(23.6)$ & $14 / 59(23.7)$ & $6 / 56(10.7)$ \\
\hline
\end{tabular}

Values given as number (percentage) unless otherwise indicated.

*Nineteen participants did not have information on the diagnosis or prescription written by the healthcare professional in their records and records for six patients were missing, so these individuals were excluded from the analyses.

the odds ratio increased to $2.7(95 \%$ CI 1.1 to 6.5 ; $\mathrm{P}=0.03)$

Judged against the test result available to the healthcare professional rather than the double read slide, in the microscopy setting there was no clinically relevant difference in the overall prescription of either antimalarials or broad spectrum antibiotics between the rapid diagnostic test arm and the microscopy arm. The similarities between the two study arms held whether test results available to healthcare professionals were positive or negative and in all age groups (tables 3 and 4). Of those patients who had malaria according to the test results available to healthcare professionals, $98.2 \%$ (587/598) in the rapid diagnostic test arm and $98.3 \%(565 / 575)$ in the microscopy arm were treated. Only $1.2 \%(11 / 598)$ of those in the rapid diagnostic test arm with malaria were not treated compared with $1.7 \%(10 / 575)$ in the microscopy arm, and all these individuals were all aged more than 15 years. This finding suggests that rapid diagnostic tests did not lead to underprescription of antimalarials as compared with microscopy. Of the 1184 patients in the rapid diagnostic test group given an antimalarial, in $50.4 \%$ (597 cases) of participants the prescriber had recorded that the result was negative.

By contrast, when prescribing in the clinical setting was compared with the healthcare professionals' interpretation of the rapid diagnostic test, there were still significant differences in the prescription of antimalarials and antibiotics between the rapid diagnostic test and clinical diagnosis arms. A total of 92.8\% (1598/ 1723) of patients in the clinical diagnosis arm were prescribed antimalarials compared with 70.0\% (1204/ $1719)$ of patients in the rapid diagnostic test arm (OR $0.18,95 \%$ CI 0.15 to $0.22 ; \mathrm{P}<0.001$; table 3$)$. Prescribing of antibiotics was $22.3 \%(384 / 1723)$ in the clinical diagnosis arm and 26.6\% (457/1719) the rapid diagnostic test arm (OR 1.3, 95\% CI 1.08 to 1.5 ;
$\mathrm{P}=0.003)$. Healthcare professionals in the clinical setting responded more logically to positive and negative rapid diagnostic test results available to them than did professionals in the microscopy setting: the former were less likely to prescribe an antimalarial to a patient who had a negative test result. Of the 1204 people in the rapid diagnostic test group given an antimalarial, $41.6 \%$ (501 cases) had a negative result on the test.

We also compared participants who had a fever or history of fever with those who did not. In the fever group, 51\% (560/1098) in the clinical diagnosis arm and $70 \%(761 / 1092)$ in the rapid diagnostic test arm were correctly prescribed antimalarials $(\mathrm{P}<0.001)$. In the group without fever or history of fever, 26\% (164/ $623)$ in the clinical diagnosis arm and $57 \%(361 / 628)$ in the rapid diagnostic test arm were correctly prescribed antimalarials. Rapid diagnostic tests, therefore, made a clinically and statistically significant difference in the correct prescription of antimalarials in both febrile and non-febrile patients.

A further analysis restricted to children under 5 years of age was undertaken. In the microscopy setting, 59\% (234/569) of children randomised to microscopy were correctly prescribed antimalarials, compared with $60 \%$ $(345 / 570)$ of children randomised to rapid diagnostic tests (OR 1.07, 95\% CI 0.8 to $2.4 ; \mathrm{P}=0.57)$. In the clinical setting, $67 \%(348 / 520)$ of children randomised to clinical diagnosis were correctly prescribed antimalarials, compared with $60 \%(225 / 570)$ randomised to rapid diagnostic tests (OR 1.7, 95\% CI 1.3 to 2.2; $\mathrm{P}<0.001)$. The impact of antibiotic prescribing has the potential to have an effect on children less than 1 year of age. In the microscopy setting, 40.2\% (35/87) of children under 1 year of age in the microscopy arm and $40.6 \%(41 / 101)$ in the rapid diagnostic test arm were prescribed an antibiotic (OR 1.01, 95\% CI 0.57 to 1.8). In the clinical setting, $29.3 \%$ (17/58) of children under 1 year of age in the clinical arm and $41.0 \%$ 
Table 5 | Incorrect prescription* of antimalarials in response to the result of rapid diagnostic tests over time

\begin{tabular}{lcc} 
& $\begin{array}{c}\text { Health centre } \\
\text { with microscopy }\end{array}$ & $\begin{array}{c}\text { Health facilities } \\
\text { with no microscopy }\end{array}$ \\
\hline Time period 1 $†$ & $248 / 531(46.8)$ & $103 / 347(29.6)$ \\
\hline Time period 2 $†$ & $45 / 186(24.2)$ & $48 / 274(17.5)$ \\
\hline Time period 3† & $76 / 368(20.7)$ & $59 / 256(23.1)$ \\
\hline Time period 4 & $149 / 591(25.2)$ & $197 / 570(34.6)$ \\
\hline Time period 5 & $91 / 223(40.8)$ & $97 / 279(34.8)$ \\
\hline
\end{tabular}

Values given as number (percentage) unless otherwise indicated.

*Incorrect prescription defined as antimalarials given to those with a

negative result on the rapid diagnostic test or withheld from those with a positive result on the rapid diagnostic test.

tEach time period was around 100 days.

$(25 / 61)$ in the rapid diagnostic test arm were prescribed an antibiotic (OR 1.67, 95\% CI 0.8 to 3.6).

Judged against the results of the double read research slides, the operational sensitivity of the rapid diagnostic tests was $86.9 \%(433 / 498)$ in the microscopy setting and $93.1 \%(606 / 651)$ in the clinical setting; the specificity of rapid diagnostic tests was 88\% (1242/1406) and $90.1 \%$ (969/1076), respectively. By contrast, the operational sensitivity of local microscopy used by healthcare professionals was $61.6 \%(316 / 513)$ and the specificity $81 \%(1033 / 1395)$ judged against the double read research slides. Sensitivity and specificity of rapid diagnostic tests were therefore both higher than routine microscopy under operational conditions.

By day 28, 10 patients in the trial had died: all were over 5 years of age. One child aged 11 years randomised to microscopy died after reporting extreme pain in the leg. The other nine deaths were in adults, four of whom were individuals aged more than 60 years. In the clinical setting, four people in the clinical arm and none in the rapid diagnostic test arm died. In the microscopy setting, two patients in the microscopy arm and four in the rapid diagnostic test arm died. Malaria was mentioned as a possible cause of death in two patients; one was 72 and the other 80 years old. One of these individuals had been treated with artemisinin based combination therapy. In the clinical setting, $266(15.9 \%)$ patients randomised to rapid diagnostic tests and $280(16.8 \%)$ randomised to clinical treatment sought further treatment of any sort.

Some studies have suggested that initial poor response to new diagnostic methods improves with time. Our study shows that in both study settings there was an initial improvement in the correct prescription of antimalarials after the first three months, but that this was not sustained (table 5).

\section{DISCUSSION}

In common with a similar study in East Africa, in this West African setting rapid diagnostic tests for malaria had no impact on the prescription of antimalarials in clinics where microscopy was available. Healthcare professionals treated the results from rapid diagnostic tests the same way as those from microscopy, often ignoring negative results and prescribing antimalarials regardless; the non-significant trend towards lower prescription of antimalarials to patients whose double read research slide was negative was because the operational sensitivity of rapid diagnostic tests was higher than that for routine microscopy.

On the other hand, in clinics where microscopy was not available and normal practice was presumptive treatment, which is typical of the majority of clinics in sub-Saharan Africa, providing rapid diagnostic tests had a clinically important impact on reducing overprescription of antimalarials. The use of rapid diagnostic tests also improved the targeting of antibiotics to patients who did not have malaria according to their double read research slide. There was no difference between the rapid diagnostic test arm and the clinical diagnosis arm in the proportions of cases of confirmed malaria that were not treated, nor evidence that introducing antimalarial treatment targeted by rapid diagnostic tests where treatment is presumptive increased mortality in children or care seeking 28 days later. Despite these encouraging results in the clinical setting, a substantial proportion of the artemisinin based combination therapy prescribed when healthcare professionals had requested a test was still to patients with a negative test result. Simply deploying rapid diagnostic tests with a limited training package is unlikely to lead to them having their full potential impact even in settings where microscopy is not available.

Major efforts are being made by the Global Fund and other organisations to increase the availability and lower the cost of antimalarials. ${ }^{20}$ The public health impact of these endeavours could be substantially undermined in West Africa, where a large proportion of the world's deaths from falciparum malaria occur, unless better targeting can be achieved. This is made more urgent by the fact that malaria makes up a decreasing proportion of febrile illness in some areas. ${ }^{8}$ The emerging resistance to artemisinin based regimens in Asia, from where previous antimalarial drug resistance has spread, probably makes it more likely that indiscriminate use of antimalarials will speed up the spread of drug resistance. ${ }^{21}$ Giving antimalarials to people who do not have malaria has no positive effect, and many alternative causes of febrile illness are potentially being missed as a result of overdiagnosis.

\section{Comparison with other studies}

Attempts to change practice in West Africa by implementing rapid diagnostic tests have been disappointing, with more than $80 \%$ of patients whose rapid diagnostic test was negative prescribed antimalarials in one study. ${ }^{22}$ Changing the behaviour of prescribers can be difficult in any setting where healthcare professionals are used to a particular prescribing pattern for a common syndrome, ${ }^{23}$ as the prolonged efforts to reduce unnecessary antibiotic prescribing in the UK have demonstrated. The reasons malaria is overdiagnosed are complex. ${ }^{24}$

The disappointing results in the microscopy setting are consistent with studies from East Africa. ${ }^{12}{ }^{13}$ Microscopy is more cost effective than rapid diagnostic tests 


\section{WHAT IS ALREADY KNOWN ON THE TOPIC}

Malaria is massively overdiagnosed in Africa, but at the same time true cases being are missed

In East Africa, introducing rapid diagnostic tests for malaria in clinics where microscopy is available has had a disappointing effect on prescribing in many settings

Most people in Africa seek care in peripheral clinics where microscopy is not available

\section{WHAT THIS STUDY ADDS}

In West Africa, the use of rapid diagnostic tests substantially reduced the overprescription of antimalarials in settings where malaria was diagnosed clinically (without microscopy, the most common situation)

Rapid diagnostic tests also led to better targeting of antibiotics in settings where microscopy was not available

Rapid diagnostic tests had limited impact on prescription of antimalarials or antibiotics in settings with microscopy facilities before is not risk-free in settings where the prevalence of blood borne viruses is relatively high. Additionally, although it is logical to assume that improving targeting of antibiotics to children who do not have malaria according to their research laboratory slide should have an impact on reducing subsequent severe bacterial illness, ${ }^{28}$ direct evidence for this is currently lacking.

\section{Limitations of the study}

The major weakness of all studies of patient and prescriber behaviour are that behaviour cannot automatically be generalised from one setting to another. This study is no exception, although it is reassuring scientifically, if not in public health terms, that our results are consistent with data from East Africa that show a limited impact of rapid diagnostic tests where microscopy exists. This agreement strengthens the likelihood that the positive impact we recorded in a community setting is in fact a real and generalisable effect. Additionally, there is always the risk that being in a trial will itself change the behaviour of healthcare professionals (the Hawthorne effect).

Much of the difference between previous studies has been assumed to be due to different training packages and diagnostic settings; in this study, prescribers in both the clinical setting and the microscopy setting were given identical training together, used the same test, and were in the same catchment area. Although residual differences in both caseload and prescriber behaviour will exist, the impact of rapid diagnostic tests was very different in the two settings. In general, the operational effectiveness of any intervention when used in normal practice is lower than that found in research studies, even in pragmatic trials.

\section{Conclusion}

The role of rapid diagnostic tests for the treatment of malaria is the subject of considerable debate. ${ }^{2930}$ This study provides evidence to support exploring the deployment of rapid diagnostic tests for malaria in West Africa in settings where microscopy is not available. The results also show that where microscopy is available, rapid diagnostic tests are unlikely to have more than a marginal impact on existing prescriber behaviour if deployed with a pragmatic training package.

Using rapid diagnostic tests is, however, likely to achieve its greatest impact only if accompanied by training and supervision tailored to the users and informed by social science studies. Although the impact of rapid diagnostic tests on prescriber behaviour in settings where diagnosis is currently syndromic (based on symptoms) is encouraging, for malaria a substantial proportion of negative tests by any technique are still ignored. This problem will need to be tackled if treatment according to rapid diagnostic tests is to be more effective and cost effective than presumptive treatment. While we acknowledge the limited generalisability, our study provides support for the deployment of rapid diagnostic tests in peripheral settings where microscopy is not available. 
We thank Constance Bart-Plange, of the National Malaria Control Programme, and Brian Greenwood for their support, and the staff in the health facilities for their critical role in identifying and recruiting patients for the study. Above all, we thank the patients who agreed to participate. Contributors: The study was conceived and designed by EKA and CJMW with contributions from JG. The study was undertaken by EKA, SN-B, ME, $\mathrm{SA}$, and AAQ. Data were analysed by EKA and CJMW. All authors commented on the manuscript, which was drafted by EKA and CJMW. EKA is the guarantor

Funding: This study was supported by the Gates Malaria Partnership and the ACT Consortium, with funds from the Bill \& Melinda Gates

Foundation. The sponsor and funder had no part in the design, conduct, analysis, or interpretation of the trial.

Competing interests: All authors have completed the Unified Competing Interest form at www.icmje.org/coi_disclosure.pdf (available on request from the corresponding author) and declare (1) CJMW has received research funding for investigator initiated research from Pfizer and GlaxoSmithKline; (2) No financial relationships with commercial entities that might have an interest in the submitted work; (3) No spouses, partners, or children with relationships with commercial entities that might have an interest in the submitted work; and (4) No non-financial interests that may be relevant to the submitted work.

Ethical approval: Ethical approval was granted by the ethics committees of the Ghana Health Service and the London School of Hygiene \& Tropical Medicine.

Data sharing: The database for this trial is available from the authors for research within the limits of the ethical approval for the trial.

1 Zurovac D, Ndhlovu M, Rowe AK, Hamer DH, Thea DM, Snow RW. Treatment of paediatric malaria during a period of drug transition to artemether-lumefantrine in Zambia: cross sectional study. BMJ 2005;331:734.

2 Amexo M, Tolhurst R, Barnish G, Bates I. Malaria misdiagnosis: effects on the poor and vulnerable. Lancet 2004;364:1896-8.

3 Nankabirwa J, Zurovac D, Njogu JN, Rwakimari JB, Counihan H, Snow RW, et al. Malaria misdiagnosis in Uganda-implications for policy change. Malar J 2009;8:66.

4 Nomhwange TI, Whitty CIM. Diagnosis of malaria in children's outpatient departments in Abuja, Nigeria. Trop Doct 2009;39:90-2.

5 Berkley JA, Maitland K, Mwangi I, Ngetsa C, Mwarumba S, Lowe BS, et al. Use of clinical syndromes to target antibiotic prescribing in seriously ill children in malaria endemic area: observational study. BMJ 2005;330:995.

6 Brent Al, Ahmed I, Ndiritu M, Lewa P, Ngetsa C, Lowe B, et al. Incidence of clinically significant bacteraemia in children who present to hospital in Kenya: community-based observational study. Lancet 2006;367:482-8.

7 Wiseman V, Kim M, Mutabingwa TK, Whitty CJM. Cost-effectiveness study of three antimalarial drug combinations in Tanzania. PLoS Med 2006;3:e373.

8 Ceesay SJ, Casals-Pascual C, Erskine J, Anya SE, Duah NO, Fulford AJ, et al. Changes in malaria indices between 1999 and 2007 in The Gambia: a retrospective analysis. Lancet 2008;372:1545-54.

9 Shillcutt S, Morel C, Goodman C, Coleman P, Bell D, Whitty CJM, et al. Cost-effectiveness of malaria diagnostic methods in sub-Saharan Africa in an era of combination therapy. Bull World Health Organ 2008;86:101-10.

10 World Health Organization. Malaria rapid diagnostic test performance: results of $\mathrm{WHO}$ product testing of malaria rapid diagnostic tests: round 1 (2008). World Health Organization, 2009.

11 Hopkins H, Kambale W, Kamya MR, Staedke SG, Dorsey G, Rosenthal PJ. Comparison of HRP2- and pLDH-based rapid diagnostic tests for malaria with longitudinal follow up in Kampala, Uganda. Am J Trop Med Hyg 2007;76:1092-7.
12 Lubell Y, Reyburn H, Mbakilwa H, Mwangi R, Chonya S, Whitty CJM, et al. The impact of response to the results of diagnostic tests for malaria: cost-benefit analysis. BMJ 2008;336:202-5.

13 Reyburn H, Mbakilwa H, Mwangi R, Mwerinde O, Olomi R, Drakeley C, et al. Rapid diagnostic tests compared with malaria microscopy for guiding outpatient treatment of febrile illness in Tanzania: randomised trial. BMJ 2007;334:403.

14 Hamer DH, Ndhlovu M, Zurovac D, Fox M, Yeboah-Antwi K, Chanda P, et al. Improved diagnostic testing and malaria treatment practices in Zambia. JAMA 2007;297:2227-31.

15 Skarbinski J, Ouma PO, Causer LM, Kariuki SK, Barnwell JW, Alaii JA et al. Effect of malaria rapid diagnostic tests on the management of uncomplicated malaria with artemether-lumefantrine in Kenya: a cluster randomized trial. Am J Trop Med Hyg 2009;80:919-26.

16 Msellem MI, Mårtensson A, Rotllant G, Bhattarai A, Strömberg J, Kahigwa $E$, et al. Influence of rapid malaria diagnostic tests on treatment and health outcome in fever patients, Zanzibar: a crossover validation study. PLoS Med 2009;6:e1000070.

17 Ssekabira U, Bukirwa H, Hopkins H, Namagembe A, Weaver MR, Sebuyira LM, et al. Improved malaria case management after integrated team-based training of health care workers in Uganda. Am J Trop Med Hyg 2008;79:826-33.

18 Valéa I, Tinto H, Nikiema M, Yamuah L, Rouamba N, Drabo M, et al. Performance of OptiMAL-IT compared to microscopy, for malaria detection in Burkina Faso. Trop Med Int Health 2009;14:338-40.

19 Ansah EK, Narh-Bana S, Asiamah S, Dzordzordzi V, Biantey K, Dickson K, et al. Effect of removing direct payment for health care on utilisation and health outcomes in Ghanaian children: a randomised controlled trial. PLoS Med 2009;6:e1000007.

20 The Global Fund. Affordable medicines facility—malaria. 2009. www. theglobalfund.org/en/amfm/.

21 Dondorp AM, Nosten F, Yi P, Das D, Phyo AP, Tarning J, et al. Artemisinin resistance in Plasmodium falciparum malaria. $N$ Engl J Med 2009;361:455-67.

22 Bisoffi Z, Sirima BS, Angheben A, Lodesani C, Gobbi F, Tinto H, et al. Rapid malaria diagnostic tests vs clinical management of malaria in rural Burkina Faso: safety and effect on clinical decisions. A randomized trial. Trop Med Int Health 2009; published online 14 February.

23 Rowe AK, Onikpo F, Lama M, Osterholt DM, Rowe SY, Deming MS. A multifaceted intervention to improve health worker adherence to integrated management of childhood illness guidelines in Benin. Am J Public Health 2009;99:837-46.

24 Chandler $\mathrm{Cl}$, Jones C, Boniface G, Juma K, Reyburn H, Whitty CJM Guidelines and mindlines: why do clinical staff over-diagnose malaria in Tanzania? A qualitative study. Malar / 2008;7:53.

25 Mundy Cl, Bates I, Nkhoma W, Floyd K, Kadewele G, Ngwira M, et al. The operation, quality and costs of a district hospital laboratory service in Malawi. Trans R Soc Trop Med Hyg 2003;97:403-8.

26 Chandler $\mathrm{Cl}$, Chonya S, Boniface G, Juma K, Reyburn H, Whitty CJM. The importance of context in malaria diagnosis and treatment decisions-a quantitative analysis of observed clinical encounters in Tanzania. Trop Med Int Health 2008;13:1131-42.

27 Zurovac D, Rowe AK, Ochola SA, Noor AM, Midia B, English M, et al. Predictors of the quality of health worker treatment practices for uncomplicated malaria at government health facilities in Kenya. Int J Epidemiol 2004;33:1080-91.

28 Reyburn H, Mbatia R, Drakeley C, Carneiro I, Mwakasungula E, Mwerinde $\mathrm{O}$, et al. Overdiagnosis of malaria in patients with severe febrile illness in Tanzania: a prospective study. BMJ 2004;329:1212.

29 English M, Reyburn H, Goodman C, Snow RW. Abandoning presumptive antimalarial treatment for febrile children aged less than five years-a case of running before we can walk? PLoS Med 2009;6:e1000015.

30 D'Acremont V, Lengeler C, Mshinda H, Mtasiwa D, Tanner M, Genton B. Time to move from presumptive malaria treatment to laboratory-confirmed diagnosis and treatment in African children with fever. PLoS Med 2009 6;6:e252.

Accepted: 15 November 2009 